\title{
E47 CHOOSING OBJECTIVE FUNCTION FOR CONDITIONING ON PRODUCTION DATA
}

Ragnar Hauge, Ole Jakob Arntzen and Harald Soleng

Norwegian computing center, PO Box 114 Blindern, N-0314 Oslo, Norway

\begin{abstract}
In this paper we consider the problem of choosing an objective function, or likelihood function, when conditioning a stochastic reservoir model on production data. We argue for a pragmatic approach where the objective function also should be judged upon its predictive value. To find candidates for objective functions, we propose that the correlation structure of production data under the prior distribution should be taken into consideration. Three different objective functions are compared; two of them are based on prior correlations while the third is a weighted sum of squares. We show by a realistic example that taking advantage of the correlation structure may increase the predictive value.
\end{abstract}

\section{Introduction}

Production data can carry essential information for prediction of reservoir performance, and it is therefore important to include this data source in the reservoir modeling. That a model matches the production history does not mean that it will predict well, since many real world configurations may give the same observations. When translating to a model, some of these matches may also be lost as the model is not as rich as reality. This means that we must admit a model error, and that multiple samples are needed to give predictions consistent with the model.

In an attempt to formalize some of these aspects we assume a Bayesian stochastic reservoir model. Technically, including production data in this setting is relatively simple, as all that is needed is an extra likelihood term in the model. Conditioning the model on the production data, i.e. sampling from the posterior distribution, require an iterative algorithm. Whether the iterative algorithm converges fast enough to be of practical value will depend strongly on the likelihood term.

Whether we regard the likelihood term as a result of data measurement errors, model errors comprising errors in the reservoir description and the flow model or, as an objective function, is mathematically irrelevant. In all cases it will influence the posterior distribution by giving more weight to small errors, i.e., high likelihoods. Measurement errors are easy to model while quantifying model errors is very hard. Unfortunately, model errors are likely to be dominant. We are therefore led to a very pragmatic approach. We ask for a likelihood term that allows the algorithm to single out realizations matching the history good enough to be promising predictors. These loosely formulated criteria will be made more precise in the following.

Traditionally, a weighted quadratic distance is often used as objective function, which corresponds to the assumption of white noise. This is not likely to be a realistic model for neither the model error nor measurement errors. Introducing a correlation structure is a natural extension 
of the simple white noise model. The choice of correlation structure in the model error is not obvious. We propose objective functions derived from the correlation structure of the data under the prior distribution. If we want the model error to leave the correlation structure largely unchanged, the model error and the model must have similar correlation structures. Hence, using the same correlation structure is a natural choice if we believe the correlation structure to be captured by the model. A simulation study using a complex reservoir model based on a realworld reservoir is carried out to see the effects.

\section{Reservoir description and model}

The modeled reservoir segment consists of the North Sea Brent group, containing the Oseberg, Rannoch, Etive, Ness and Tarbert formations. Only the Etive and Ness formations give significant contribution to the initial reserves, so only these formations are modeled stochastically. The rest of the formations are modeled deterministically, and are therefore identical both in our synthetic true reservoir and all our sampled reservoirs.

The Oseberg formation is 55-65 meters thick, followed by a 9-12 meter thick Rannoch. The Etive formation is 10-13 meters thick, and is modeled directly on the petrophysical level, using a Gaussian field for porosity, and a log-Gaussian field for permeability. For the Ness formation, a

fluvial object model is used to model facies, and petrophysics are then added as for the Etive, but conditional on facies, with poor quality where the facies model does not indicate sand.

Furthermore, the Ness zone has been split in two, lower and upper Ness, with no pressure communication between them. Lower Ness is assumed to be in communication with Etive. Total thickness of the Ness formation is 35-45 meter. Finally, there is a 5-7 meter thick Tarbert with no pressure communication with the rest of the field. The segment is approximately 1 by $3 \mathrm{~km}$.

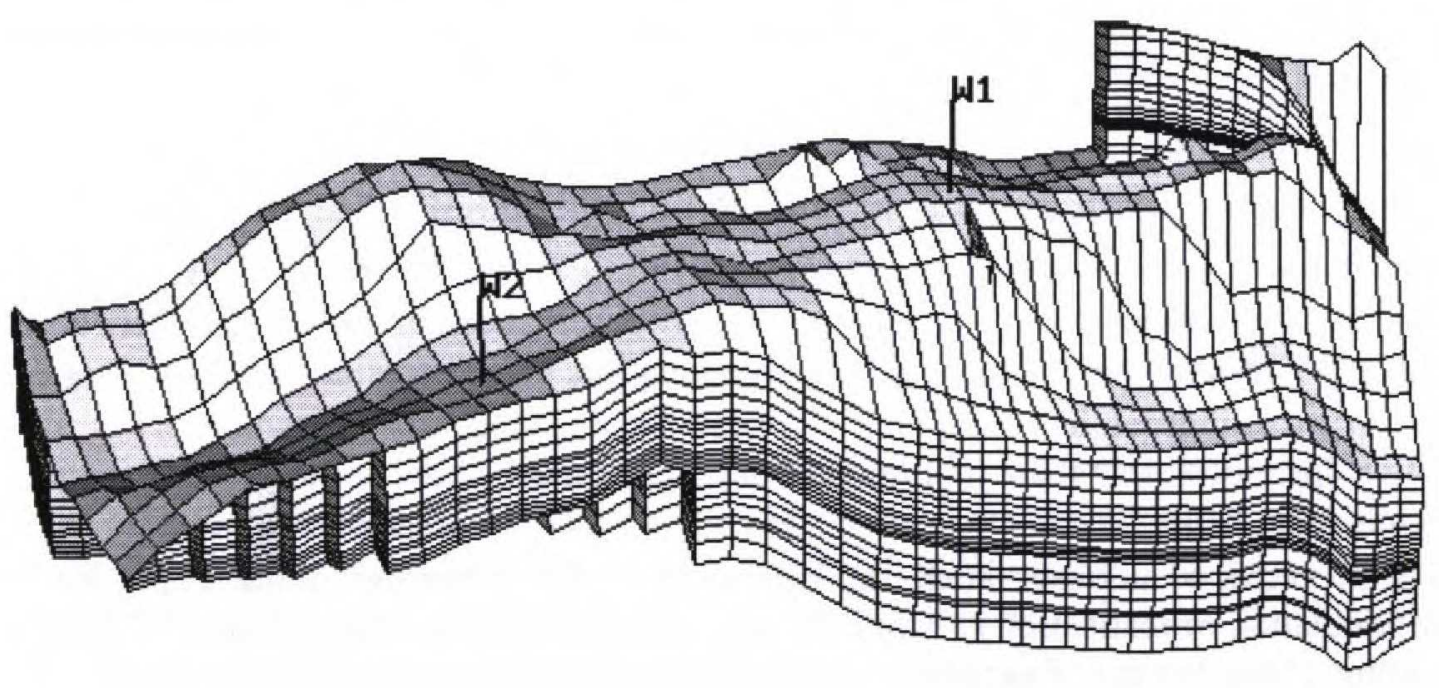

Figure 1: The simulation grid layout with well locations.

The reservoir contains light oil with dissolved gas. Initially there is no free gas. The boiling point is 176 bars while the initial pressure is above 300 bars. Maintaining the pressure above the boiling point to accelerate production is a challenge given our assumptions on the facilities.

The segment in our model has no communication with the surrounding field. Two wells have been drilled in the segment. The first well, W1, starts production from Oseberg and Etive at time $\mathrm{t}=0$. At time $\mathrm{t}=34$ (measured in months), it also starts producing from upper Ness, and 6 months later, production from lower Ness is added. This well, which is the main producer, operates with 
a maximum rate of 1000 standard $\mathrm{m}^{3}\left(\mathrm{Sm}^{3}\right)$ and a minimum bottom-hole pressure of 150 bars. The second well, W2, starts as a producer from upper Ness at time $t=27$, and production from Tarbert is added 5 months later. It produces a specified total rate in reservoir $\mathrm{m}^{3}\left(\mathrm{Rm}^{3}\right)$. At time $t=42$, it is changed to an upper Ness injector to maintain pressure above the boiling point there. The inter-well distance is $1200 \mathrm{~m}$. The statistical model is conditioned on well log data. These are facies and petrophysics logs and constitute the well data term $d_{w}$ in the model.

The geomodel grid resolution is $47 \times 106 \times 110$ cells, which is upscaled to a $46 \times 9 \times 20$ simulation grid. Upscaled porosities and horizontal permeabilities are arithmetic averages, while upscaled vertical permeabilities are harmonic averages. The production data used for conditioning are oil production rates from the two wells, observed approximately once a month, the water cut in the wells, and a few well block pressure observations. There are 222 observations in the observation vector, which is denoted $d_{p}$.

As mentioned, we use a Bayesian stochastic model, which is given by $\pi\left(r \mid d_{p}, d_{w}\right) \propto f_{r}\left(r \mid d_{w}\right) f_{d}\left(d_{p} \mid r\right)$

where $\pi$ denotes the posterior likelihood, $f_{r}$ denotes the likelihood for the reservoir vector $r, f_{d}$ denotes the likelihood for the production data, and $d_{w}$ and $d_{p}$ are vectors containing well data and production data respectively. Contained in $r$ are facies, petrophysics, and transmissibility multipliers. Since our focus is on the conditioning on production data, the distribution of $r$ conditioned on well data is regarded as our prior. The stochastic reservoir model described above defines this prior. The second term on the right is the likelihood for production given reservoir, which is our main concern. This likelihood is the exponential of the negative objective function.

Using a standard squared sum objective function gives the expression

$$
f\left(d_{p} \mid r\right)=\exp \left(-\frac{1}{2} \sum_{t=0}^{T} \sum_{i=1}^{N}\left(\frac{d_{i}^{t}-h_{i}^{t}(r)}{\sigma_{i}^{t}}\right)^{2}\right)
$$

where $d_{i}^{t}$ is production observation $i$ at time $t$ and $h_{i}^{t}(r)$ is the corresponding output from the reservoir simulator given realization $r$. The denominator $\sigma_{i}^{t}$ is the standard deviation for the error in the corresponding observation. At each time step, $N$ observations are made, and there are $T$ time steps. If we instead use a correlated objective function, the expression becomes

$$
f\left(d_{p} \mid r\right)=\exp \left(-\frac{1}{2}(d-h(r)) \Sigma^{-1}(d-h(r))\right)
$$

where $d$ is the vector of all observations, and $h(r)$ is the vector of corresponding output from the reservoir simulation of realization $r$. A full covariance matrix $\Sigma$ is included, giving full freedom for correlations between any observations independent of time and type of observation.

We sample from the posterior by a Markov Chain Monte Carlo (MCMC) algorithm, and hence the normalizing constant is not needed. The MCMC algorithm is an iterative algorithm that works by proposing realizations and then accepts or rejects them based on likelihood. New proposals are based on the last accepted realization. In our implementation, we allow both large changes, which have low acceptance probabilities but allows jumping between posterior modes, and small changes, which have high acceptance probability but only explore the state space locally. The large changes consist of drawing a new realization for one of the modeled zones (upper or lower Ness, or Etive), while the small changes only do local perturbations, such as moving a few channels. 


\section{Simulation study}

Initially, we generated 200 realizations from the prior distribution. The production results from these are shown in Figure 2.
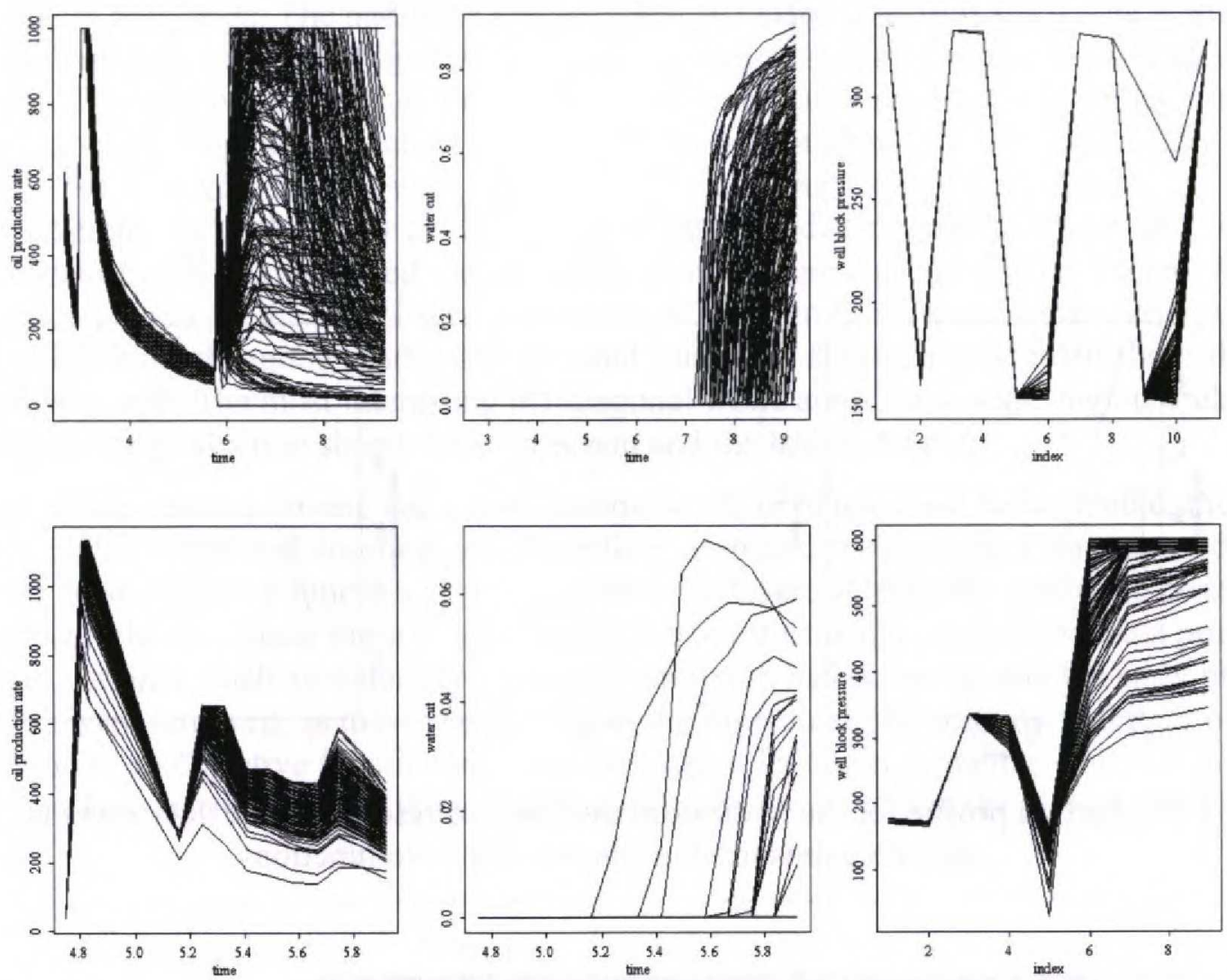

Figure 2: Production from 200 prior realizations. The top row is for W1, the bottom row for W2. Oil production rate is shown in the first column, water cut in the second column, and selected well block pressure data in the third. The time unit is years, volume unit is $\mathrm{Sm}^{3}$ and pressure unit is bars. Note the different time scales.

As seen from the figure, there is not much variation in the production in W1 until W2 starts injecting. The communication between the wells is then crucial, and depending on this, production after injection varies from zero to the target value. Since W2 is controlled by total rate, the oil rate there depends on the presence of free gas near the well. Most realizations have water breakthrough in W1; some also have breakthrough in W2 in the short time that well is used as producer. The pressure variation in W1 is also small, with one realization standing out. In W2, there is large variation in some pressure observations.

From these 200 realizations, one was selected as our reservoir to be matched. Its production profile is shown in Figure 3. This realization has weak communication between the wells, giving the production in W1 a boost after W2 starts injecting, but not enough to reach the target. There is a late water breakthrough in W1, but no breakthrough in W2.

The purpose of this study is to compare different objective functions, with some of them depending on the correlation structure of the prior production. The estimate of this correlation structure, based on the 200 realizations, is shown as a plot of the correlation matrix in Figure 4. 

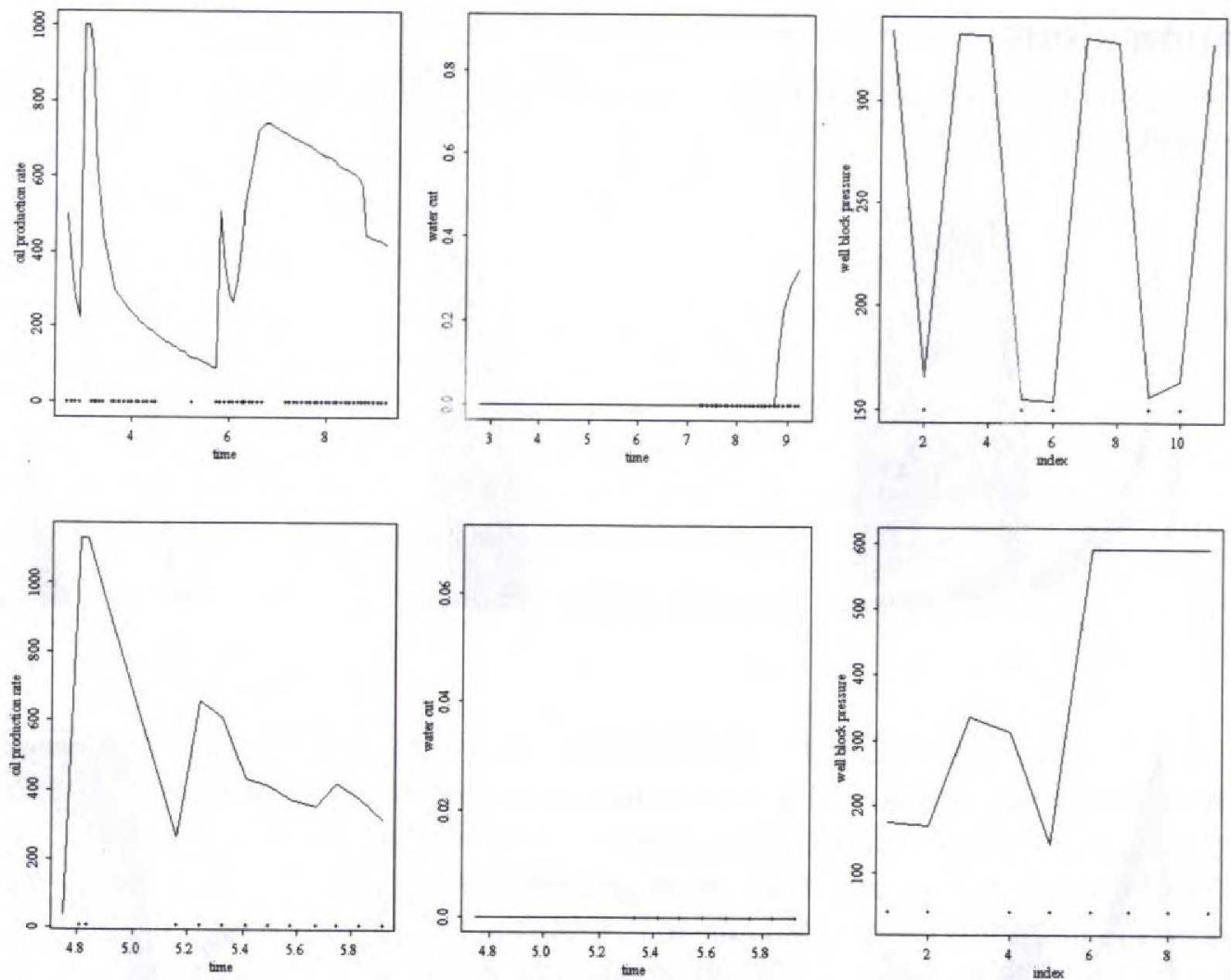

Figure 3: Production profile for the realization used as true reservoir. The dots along the bottom indicate points used in filtered objective functions.

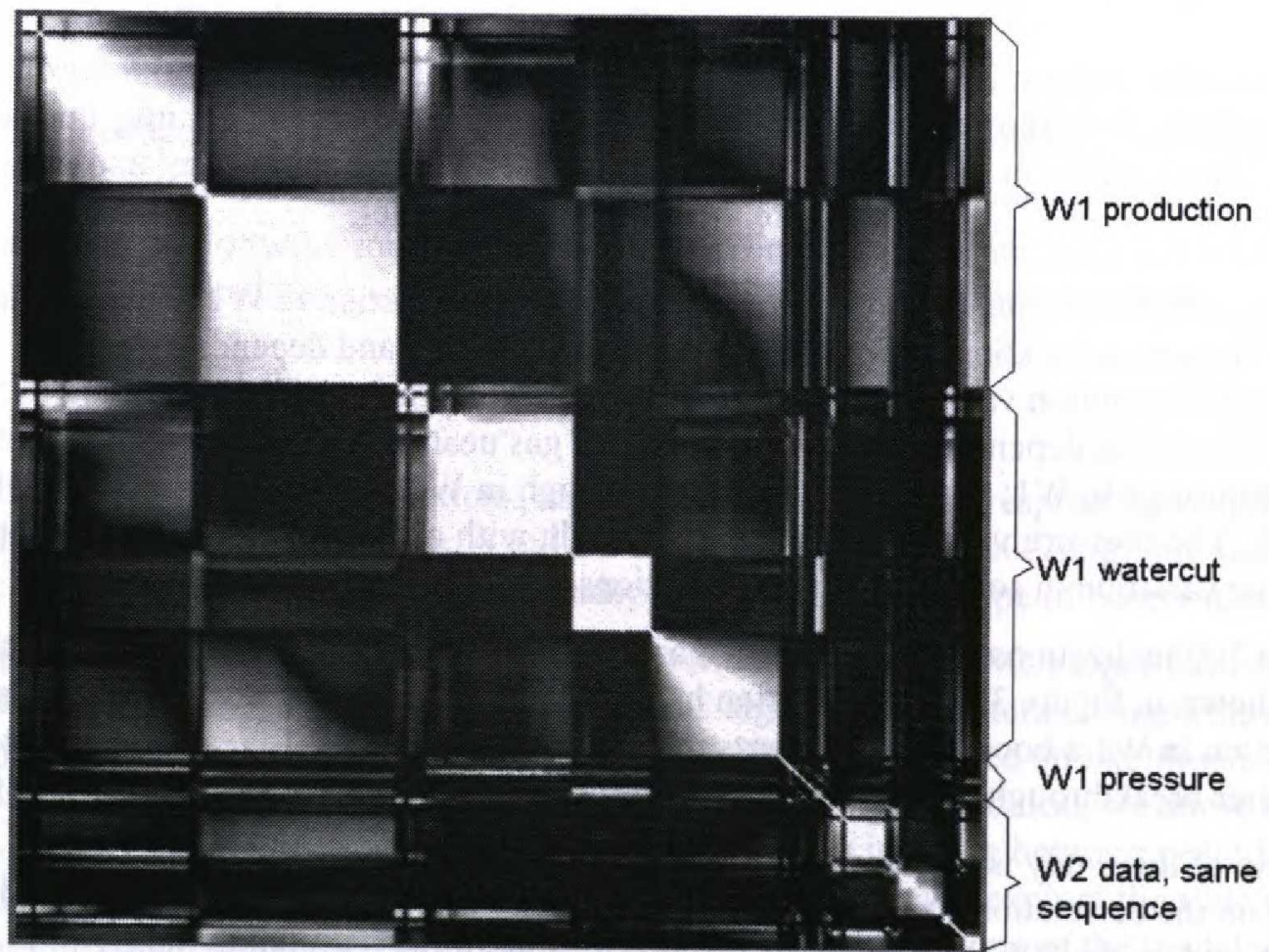

Figure 4: Visualization of the correlation matrix. Light values correspond to high correlations (either positive or negative). 
The data are arranged in the matrix as oil rate data from W1 (89 observations), followed by water cut ( 87 observations) and pressure data ( 11 observations) from the same well. This sequence is then repeated for W2, with 13, 13 and 9 observations respectively. The first large white square in the upper left corner shows that the production after leaving plateau for the first time is highly correlated. The pattern is broken when W2 starts injecting, but a new white square along the diagonal shows that all production data after injection are also highly correlated with each other. The next two squares are only related to numerical noise from the simulator, as the underlying observation here is water cut and water breakthrough has not occurred in any realization yet. The third square, with darker areas towards the lower left and upper right shows that the development of the water cut is also highly correlated, especially from the time when most realizations have experienced breakthrough. The pressure data seem uncorrelated. Finally, a correlation pattern for the few observations from W2 is seen in the lower right corner, with high correlations both among production data and water cut, although most realizations do not have breakthrough. The most interesting off-diagonal white areas show some high correlations in W1 between the production shortly after injection and the late water cut.

A full covariance matrix having these correlations would in practice not be invertible; there are too many highly correlated observations. Therefore, a filtered subset of the data was used for the correlated noise objective function. The data points used were selected by removing observations with high correlations, since these do not contain much information. In addition, data points with very small variance, such as water cut before breakthrough had occurred, and the early plateau production were removed, as these remain almost the same in all realizations, and their only contribution in an objective function would be to make it less discriminating. The data points actually used as observations are indicated by stars along the bottom in the plots in Figure 3 .
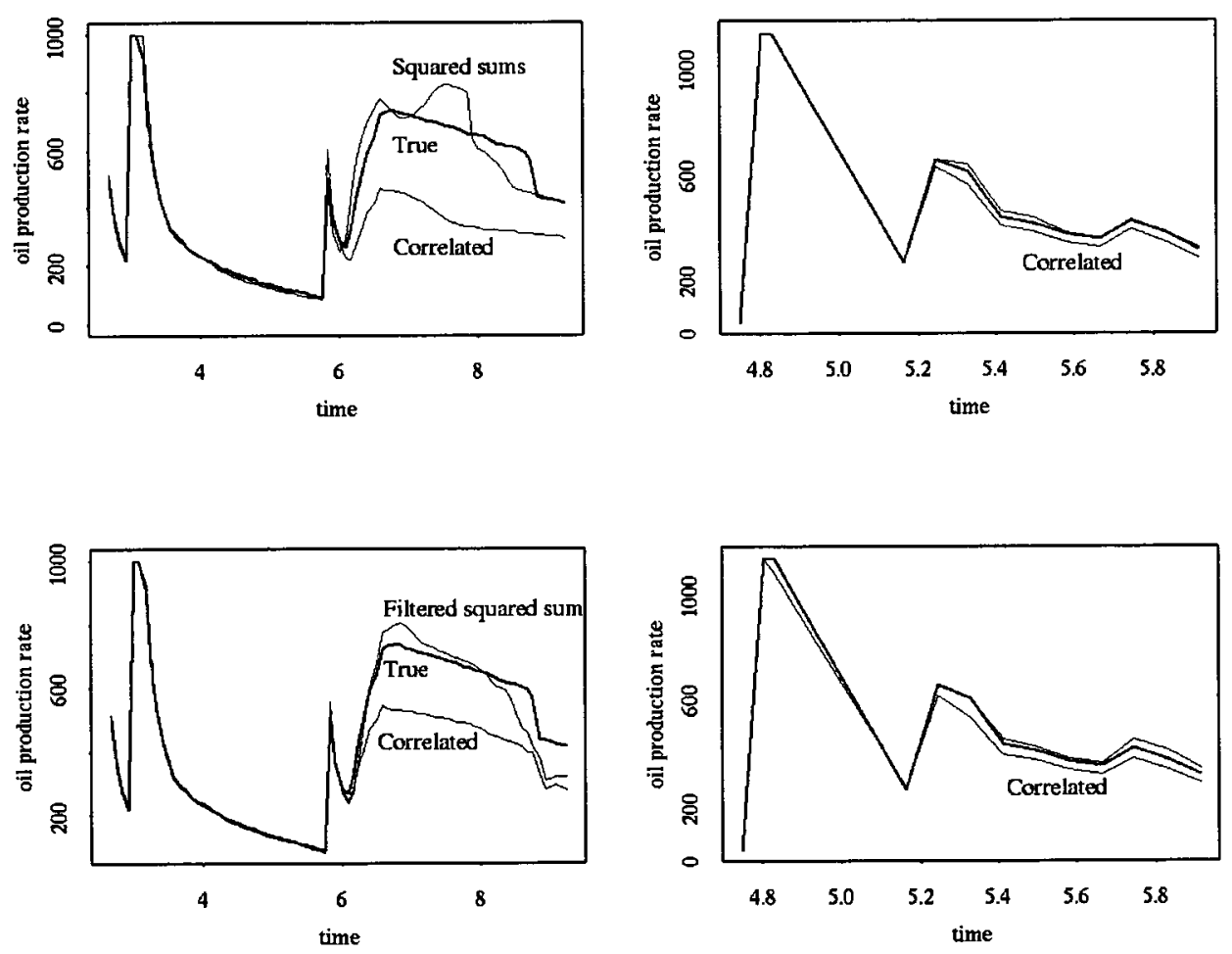

Figure 5: The top row shows true production along with the curves from the prior sample said to be closest by the three objective functions (only oil rates considered). The bottom row shows the true curve along with posterior samples from two objective functions. Only oil rates are shown, with $\mathrm{W} 1$ to the left. In the top row, the full and filtered squared sums prefer the same realization. 
We tested three different objective functions. One used a full sum of squares, one used a covariance matrix over the observations remaining after filtering, and one used a sum of squares only over these observations. The estimated prior variance in each point was used as variance. This corresponds to assigning equal importance to each point in this case.

The preferences of the different objective functions can be seen in the production curves in Figure 5. This figure shows the true production in the two wells, along with preferred prior realizations and realizations from posterior. Clearly, the correlated objective function assigns importance to shape, whereas the ones based on the squared sums prefers small errors at all times.

A simple way of comparing the objective functions is to look at the correlation between the value of the objective function based on data up to time $t$ and a weighted sum of the squared difference between predicted and true production after time $t$, denoted as the squared error (SE). The prior variances are used as weights. This gives an indication of the predictive value of the objective function. The results are shown in Figure 6. As time $t$ increases, more observations are used as data, and less as prediction values, so we would expect the correlations to increase. This happens for the two squared sums objective functions after the injection starts, but the correlated objective function gives a more erratic result. The reason for this is that the correlations give larger weight to having correct shape than to having correct level.

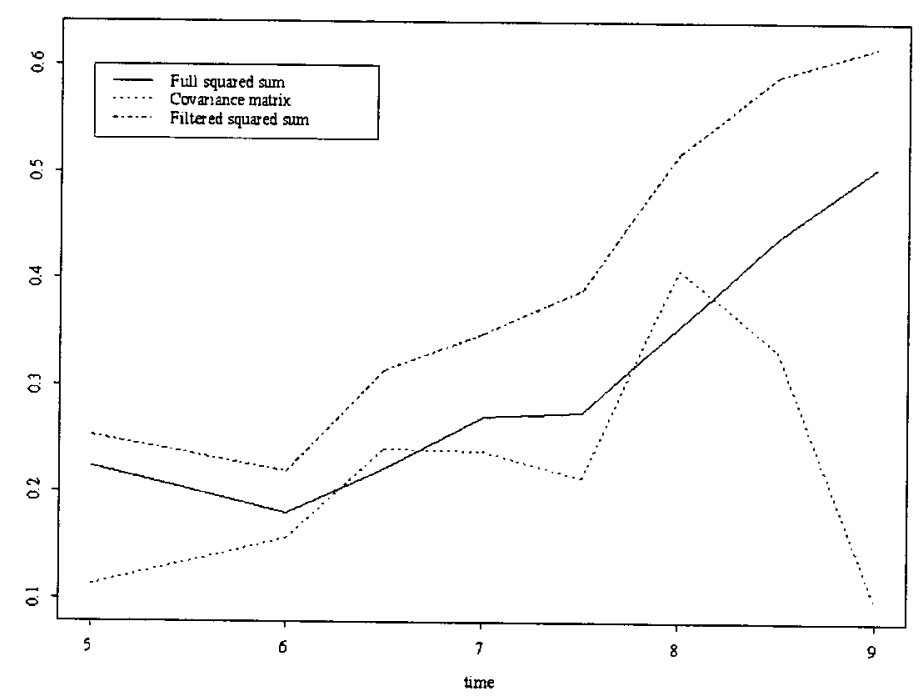

Figure 6: Correlation between objective function and prediction error as function of time of prediction.

A weakness with the correlation is that it only looks for linear relationships. Looking at the prior expected SE given the value of the objective function gives a more precise comparison. We did this by grouping values of the objective function into bins, and taking the average of the weighted SE in each bin. The results we got were very much in agreement with what we got using correlations, and we saw no tendency to non-linear relationships between objective values and prediction errors. It is clear that, for a fixed sample size, the correlation estimates are statistically more significant than the estimates of the conditioned SE.

The correlations seen in Figure 6 are all rather small. We would expect small correlations for the earliest times, since the correlation plot showed small correlations between behavior before and after injection started. That the correlation does not reach higher levels is mainly due to the water breakthrough. Our true reservoir has a late water breakthrough, and realizations that match early on tend to have earlier water breakthroughs, giving larger values for the prediction error.

$8^{\text {th }}$ European Conference on the Mathematics of Oil Recovery - Freiberg, Germany, 3 - 6 September 2002 
Another point of interest was whether the choice of objective function had any influence on the convergence of an algorithm for sampling from the posterior. Clearly, if two different objective functions have about the same predictive strength, the one giving best convergence is preferable. In order to make the comparison fair, the variances in the objective functions were scaled so that they gave the same span of values for the realizations in the sample of 200 prior realizations when extreme outliers had been removed.

Using the full squared sum gave the slowest convergence. From logs of values for the two other objective functions, the distribution of these values appears to stabilize after about 600 iterations in both cases, indicating an equal convergence speed. The acceptance ratios were approximately equal, as was the number of major changes. With this setup, the choice between the correlated and filtered objective function seems to have no influence on convergence.

\section{Concluding remarks}

We have looked at the effects of using correlation structures in the production data from the prior model when choosing objective function for conditioning on production data. The production data correlation matrix gives much information about the behavior, indicating observations that hold information about each other. Here, it showed strong correlations around the diagonal in large areas, which means that matching these observations becomes very important if correlation effects are ignored. In addition, although we did not explore that option here, the correlation matrix could be used to find observations that are highly correlated with production at some future time and give these extra weights in the objective function.

Three different objective functions were compared with respect to predictive strength and convergence. Two of these were based on the prior correlation matrix. Using a filtered sum of squares, where some of the highly correlated observations were removed, was a success both regarding prediction strength and convergence rate. Without the filtering, the prediction strength was less, and convergence slower. Using the covariances explicitly in the objective function was not so successful. This objective function seemed to put too much emphasis on shape, while ignoring level. The corresponding squared errors in the prediction were therefore large. Convergence speed was as for the filtered sum of squares.

We would like to thank our colleague Sigurd Sannan for his participation in setting up the simulation and sampling framework.

[1] Aziz K., Settari A., Petroleum Reservoir Simulation, Applied Science Publishers LTD, 1979.

[2] Floris F. J.T., Bush M. D., Cuypers M., Roggero F., Syversveen A. R., Methods for quantifying the uncertainty in production forecasts: a comparative study, Petroleum Geoscience, vol. 7, pp. S87-S96, Special Issue, 2001.

[3] Holden L., Hauge R., Skare Ø., Skorstad A., Modelling of fluvial reservoirs with object models, Mathematical Geology, vol. 30, pp. 473-496, July 1998.

[4] Gilks, W.R., Richardson, S., Spiegelhalter, D.J., Markov Chain Monte Carlo in practice, Chapman \& Hall, 1996. 